\title{
Metal-Organic Frameworks as an Appropriate Platform for Controlled Drug Release
}

\author{
Tahereh Azizi Vahed, Mohammad Reza Naimi-Jamal*, Leila Panahi \\ Department of Chemistry, Research Laboratory of Green Organic Synthesis \& Polymers,

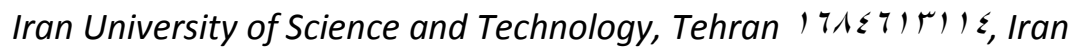 \\ Email: naimi@iust.ac.ir
}

\begin{abstract}
Herein we used MOFs as a platform for controlled drug release and reported a technique, which pharmaceutical agents are encapsulated in a zinc zeolitic imidazolate framework (ZIF- $\curlywedge$ ). In this work, metformin, an oral antihyperglycemic agent for the treatment of type II diabetes trapped inside the ZIF-A pores through a mechanochemical ball-milling technique. Due to having regular porosity and zinc ions, ZIF-A could help to better absorption of metformin. The synthesis of the MOF and the encapsulating process of the drug were performed by using a convenient solvent-free one pot technique. It was shown that with this approach, metformin is released in the stomach via a controlled manner over a longer time that leads to increases of metformin bioavailability.
\end{abstract}

Keywords: ZIF-^, Metformin, drug delivery, controlled release

\section{Introduction}

Metal-organic frameworks are a class of porous materials which are synthesized trough the self-assembly of metal ions and organic linkers. These particles have high pore volume and surface area; hence they are highly appropriate for the use as catalysts, sensors, absorbent and most recently in medicine. ${ }^{\left[{ }^{1-T}\right]}$ Recent studies illustrated that the MOFs can be promising platforms to entrap and gradual release of drugs or biomolecules. The first use of the MOFs as a drug delivery platform was carried out by Ferey et al in $2004{ }^{[7]}$ and then other studies proved that these particles have a high payload efficiently $(r \cdot-v \cdot \%){ }^{\left[{ }^{[}\right]}$to encapsulate the drug molecules. Among these MOFs, ZIF- $\wedge$ with a $\mathrm{pH}$-sensitive property, suitable shape and size is a non-toxic framework which has a good track record in using as drug delivery systems (DDS). ${ }^{\left[{ }^{9}, 1 \cdot\right]}$ Herein we utilized ZIF- $\wedge$ nanoparticles to encapsulate and controlled release of metformin. This method is based on a solvent free and one-pot mechanochemical reaction that the drug-loaded nanoparticles were synthesized via the ball milling a mixture of reactants such as zinc ion source (zinc acetate), r-Methylimidazole (an organic linker) and metformin as a model drug (Scheme 1). 


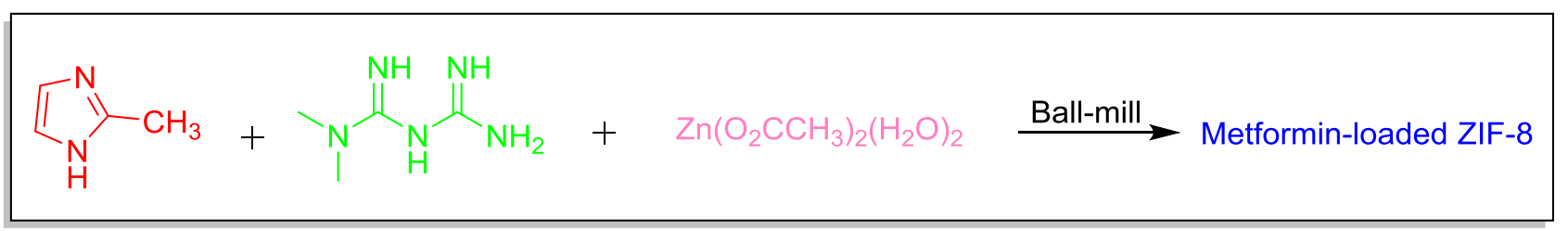

Scheme '. Synthesis of metformin-loaded ZIF-^

\section{Experimental}

\section{Materials and characterization}

In this work all chemical reagents were purchased from Merck and Sigma-Aldrich (of analytical grade) and used without further purification. Fourier transform infrared (FT-IR) spectroscopy was carried out to ensure the product attainment and the spectrum was recorded on Nicolet Magna-00. spectrometer in $\mathrm{KBr}$ pellet. UV-vis spectroscopy analysis using a Shimadzu UV-vis scanning spectrometer was carried out to monitor the drug release from the MOF.

\section{Synthesis of drug-loaded MOFs}

A mixture of zinc acetate, $r$-Methylimidazole and metformin in the ratio of $1: r: \bullet .0$ were placed in the ball milling case, after I hour mixture turned to milky dough which signified the MOF forming. Relevant peaks in the IR spectrum confirmed formation of the drug-loaded MOF which in addition to the peaks that are related to the framework, drug relevant peaks were observed in the spectrum (Figure ').

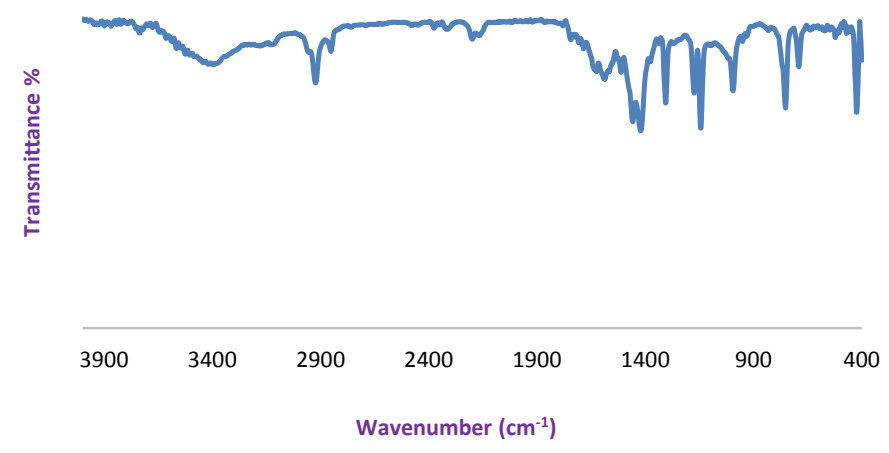

Figure I. IR spectrum of Met-loaded ZIF-A 


\section{Result and discussion}

To investigate the release procedure, a certain amount of drug-loaded ZIF- $\wedge$ was incubate in the buffered saline (PBS) under magnetic stirring at $r v{ }^{\circ} \mathrm{C}$ and in different times a small amount of the solution was analyzed by UV-vis spectroscopy. We investigated the released drug concentration in different time and the curve showed controlled release of the drug from the framework (Figure $r$ ).

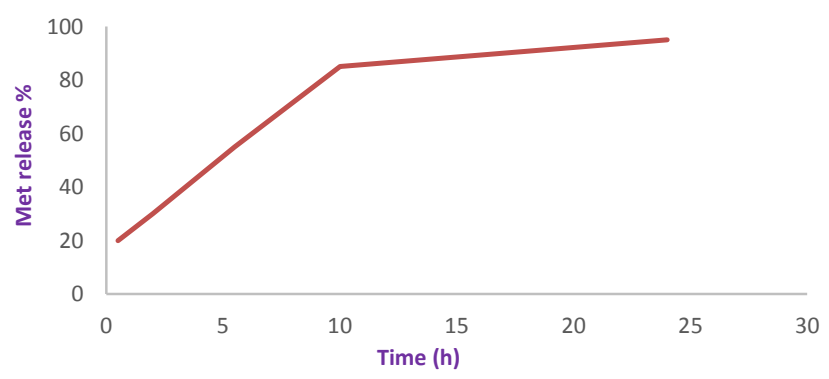

Figure $r$. Metformin release from ZIF- $\wedge$ in PBS $p H \vee . \leqslant$ at $r{ }^{\circ} \mathrm{C}$.

\section{Conclusion}

In summary, we have achieved an appropriate platform to increase the bioavailability of metformin, a high challenging drug. This process was done through a green solvent free, onepot and short time reaction that drug molecules were encapsulated in ZIF-^ particles, coincided with the nanoparticles synthesis.

\section{Acknowledgements}

The authors gratefully acknowledge the partial support from the Research Council of the Iran University of Science and Technology.

\section{References:}

1. Zhao, D., et al., Tuning the topology and functionality of metal- organic frameworks by ligand design. Accounts of chemical research, 2010. 44(2): p. 123-133.

2. Corma, A., H. García, and F. Llabrés i Xamena, Engineering metal organic frameworks for heterogeneous catalysis. Chemical Reviews, 2010. 110(8): p. 4606-4655.

3. Li, J.-R., R.J. Kuppler, and H.-C. Zhou, Selective gas adsorption and separation in metal-organic frameworks. Chemical Society Reviews, 2009. 38(5): p. 1477-1504.

4. Keskin, S. and S. Kızılel, Biomedical applications of metal organic frameworks. Industrial \& Engineering Chemistry Research, 2011. 50(4): p. 1799-1812.

5. Yang, X.L., et al., A Multiresponsive Metal-Organic Framework: Direct Chemiluminescence, Photoluminescence, and Dual Tunable Sensing Applications. Advanced Functional Materials, 2016. 26(3): p. 393-398. 
6. Kaur, R., et al., Recent advances in the photovoltaic applications of coordination polymers and metal organic frameworks. Journal of Materials Chemistry A, 2016. 4(11): p. 3991-4002.

7. Férey, G., et al., A hybrid solid with giant pores prepared by a combination of targeted chemistry, simulation, and powder diffraction. Angewandte Chemie, 2004. 116(46): p. 6456-6461.

8. Agostoni, V., et al., A "green" strategy to construct non-covalent, stable and bioactive coatings on porous MOF nanoparticles. Scientific reports, 2015. 5.

9. Zhuang, J., et al., Optimized metal-organic-framework nanospheres for drug delivery: evaluation of small-molecule encapsulation. ACS nano, 2014. 8(3): p. 2812-2819.

10. Tian, Z., X. Yao, and Y. Zhu, Simple synthesis of multifunctional zeolitic imidazolate frameworks-8/graphene oxide nanocrystals with controlled drug release and photothermal effect. Microporous and Mesoporous Materials, 2017. 237: p. 160-167. 